\title{
Searches for heavy neutrinos from $Z$ decays
}

\author{
DELPHI Collaboration
}

P. Abreu ${ }^{a}$, W. Adam ${ }^{b}$, F. Adami ${ }^{c}$, T. Adye ${ }^{d}$, T. Akesson ${ }^{\text {e }}$, G.D. Alekseev ${ }^{f}$, P. Allen ${ }^{\mathrm{g}}$, S. Almehed ${ }^{\text {e, S.J. Alvsvaag }}{ }^{\mathrm{h}}$, U. Amaldi ${ }^{1}$, E. Anassontzis ${ }^{\mathrm{j}}$, P. Antilogus ${ }^{\mathrm{k}}$, W.-D. Apel ${ }^{\ell}$, R.J. Apsimon ${ }^{\mathrm{d}}$, B. Åsman ${ }^{\mathrm{m}}$, P. Astier ${ }^{\mathrm{n}}$, J.-E. Augustin ${ }^{\circ}$, A. Augustinus ${ }^{\mathrm{p}}$, P. Baillon ${ }^{\mathrm{i}}$, P. Bambade ${ }^{\circ}$, F. Barao a , R. Barate ${ }^{\mathrm{q}}$, G. Barbiellini ${ }^{\mathrm{r}}$, D.Y. Bardin ${ }^{\mathrm{f}}$, A. Baroncelli ${ }^{\mathrm{s}}$, O. Barring e, W. Bartl ${ }^{\text {b }}$, M.J. Bates ' ${ }^{\text {, M. Battaglia }}{ }^{\text {u }}$, M. Baubillier ${ }^{\text {n }}$, K.-H. Becks ${ }^{\text {, }}$, C.J. Beeston ${ }^{t}$, M. Begalli ${ }^{\text {}}$, P. Beilliere ${ }^{\mathrm{x}}$, Yu. Belokopytov ${ }^{\mathrm{y}}$, P. Beltran ${ }^{\mathrm{z}}$, D. Benedic ${ }^{\text {aa }}$, J.M. Benlloch ${ }^{\mathrm{g}}$, M. Berggren ${ }^{\circ}$, D. Bertrand ${ }^{\mathrm{ab}}$, F. Bianchi ${ }^{\text {ac }}$, M.S. Bilenky ${ }^{\mathrm{f}}$, P. Billoir ${ }^{\mathrm{n}}$, J. Bjarne ${ }^{e}$, D. Bloch ${ }^{\text {aa }}$, S. Blyth ${ }^{t}$, V. Bocci ${ }^{\text {ad }}$, P.N. Bogolubov ${ }^{f}$, T. Bolognese ${ }^{c}$, M. Bonapart ${ }^{p}$, M. Bonesini ${ }^{\text {u }}$, W. Bonivento ", P.S.L. Booth ${ }^{\text {ae }}$, M. Boratav ${ }^{\mathrm{n}}$, P. Borgeaud ${ }^{\mathrm{c}}$, G. Borisov ${ }^{\mathrm{y}}$, H. Borner ${ }^{i}$, C. Bosio ${ }^{\text {s }}$, B. Bostjancic ${ }^{\text {i }}$, O. Botner ${ }^{\text {af }}$, B. Bouquet ${ }^{\circ}$, C. Bourdarios ${ }^{\circ}$, M. Bozzo $^{\text {w, }}$, S. Braibant ${ }^{\text {ab }}$, P. Branchini s, K.D. Brand ${ }^{a g}$, R.A. Brenner ${ }^{\text {ah }}$, C. Bricman ${ }^{\text {ab }}$, R.C.A. Brown ${ }^{\mathrm{i}}$, N. Brummer ${ }^{\text {p }}$, J.-M. Brunet ${ }^{x}$, L. Bugge ${ }^{\text {ai }}$, T. Buran ${ }^{\text {ai }}$, H. Burmeister ${ }^{\text {i }}$, J.A.M.A. Buytaert ${ }^{\text {, }}$, M. Caccia ${ }^{\text {i }, ~ M . ~ C a l v i ~}{ }^{\text {}}$, A.J. Camacho Rozas ${ }^{\text {aj }}$, A. Campion ${ }^{\text {ae }}$, T. Camporesi i, V. Canale ${ }^{\text {ad }}$, F. Cao ${ }^{\text {ab }}$, F. Carena ${ }^{i}$, L. Carroll ${ }^{\text {ae }}$, C. Caso ${ }^{\text {w }}$, E. Castelli ${ }^{\text {r }}$, M.V. Castillo Gimenez ${ }^{\text {g }}$, A. Cattai ${ }^{i}$, F.R. Cavallo ${ }^{\text {ak }}$, L. Cerrito ${ }^{\text {ad }}$, A. Chan ${ }^{\text {al }}$, M. Chapkin ${ }^{y}$, P. Charpentier ${ }^{\mathrm{i}}$, L. Chaussard ${ }^{\circ}$, P. Checchia ${ }^{\text {ag }}$, G.A. Chelkov ${ }^{f}$, L. Chevalier ${ }^{c}$, P. Chliapnikov ${ }^{y}$, V. Chorowicz ${ }^{n}$, R. Cirio ${ }^{\text {ac }}$, M.P. Clara ${ }^{\text {ac }}$, P. Collins ${ }^{t}$, J.L. Contreras ${ }^{\text {am }}$, R. Contri ${ }^{w}$, G. Cosme ${ }^{\circ}$, F. Couchot ${ }^{\circ}$, H.B. Crawley a , D. Crennell ${ }^{\text {d }}$, G. Crosetti ${ }^{\text {w }}$, M. Crozon ${ }^{x}$, J. Cuevas Maestro ${ }^{\text {aj }}$, S. Czellar ${ }^{\text {ah }}$, S. Dagoret ${ }^{\circ}$, E. Dahl-Jensen ${ }^{\text {an }}$, B. Dalmagne ${ }^{\circ}$, M. Dam ${ }^{\text {ai }}$, G. Damgaard ${ }^{\text {an }}$, G. Darbo ${ }^{\text {w }}$, E. Daubie ${ }^{\mathrm{ab}}$, P.D. Dauncey ${ }^{\mathrm{t}}$, M. Davenport ${ }^{\mathrm{i}}$, P. David ${ }^{\mathrm{n}}$, C. Defoix ${ }^{\mathrm{x}}$, D. Delikaris ${ }^{\mathrm{i}}$, S. Delorme ${ }^{\text {i }}$, P. Delpierre ${ }^{x}$, N. Demaria ${ }^{\text {ac }}$, A. De Angelis ${ }^{r}$, M. De Beer ${ }^{c}$, H. De Boeck ${ }^{\text {ab }}$, W. De Boer ${ }^{\ell}$, C. De Clercq ${ }^{\text {ab }}$, M.D.M. De Fez Laso ${ }^{g}$, N. De Groot ${ }^{p}$, C. De La Vaissiere ${ }^{\text {, }}$ B. De Lotto ${ }^{r}$, A. De Min ", H. Dijkstra ${ }^{i}$, L. Di Ciaccio ${ }^{\text {ad }}$, F. Djama ${ }^{\text {aa }}$, J. Dolbeau ${ }^{x}$, O. Doll ${ }^{\text {, }}$ M. Donszelmann ${ }^{\mathrm{p}}$, K. Doroba ${ }^{\mathrm{ao}}, \mathrm{M}$. Dracos ${ }^{\mathrm{i}}$, J. Drees ${ }^{\mathrm{v}}$, M. Dris ${ }^{\mathrm{ap}}$, Y. Dufour ${ }^{\mathrm{x}}$, W. Dulinski ${ }^{\text {aa }}$, R. Dzhelyadin ${ }^{\mathrm{y}}$, L.-O. Eek ${ }^{\text {af }}$, P.A.-M. Eerola ${ }^{\mathrm{i}}$, T. Ekelof ${ }^{\text {af }}$, G. Ekspong ${ }^{\mathrm{m}}$, A. Elliot Peisert ${ }^{\text {ag }}$, J.-P. Engel ${ }^{\text {aa }}$, D. Fassouliotis ${ }^{\text {ap }}$, M. Feindt ' ${ }^{\text {, M. Fernandez Alonso }}{ }^{\text {aj, }}$, A. Ferrer ${ }^{\mathrm{g}}$, T.A. Filippas ${ }^{\mathrm{ap}}$, A. Firestone ${ }^{\mathrm{a} \ell}$, H. Foeth ${ }^{\mathrm{i}}$, E. Fokitis ${ }^{\text {ap }}$, P. Folegati ${ }^{\mathrm{r}}$, F. Fontanelli ${ }^{\text {w }}$, K.A.J. Forbes ${ }^{\text {ac }}$, H. Forsbach ${ }^{\text {, }}$, B. Franek ${ }^{d}$, P. Frenkiel ${ }^{x}$, D.C. Fries ${ }^{\ell}$, A.G. Frodesen ${ }^{\text {h }}$, R. Fruhwirth ${ }^{\text {b }}$, F. Fulda-Quenzer ${ }^{\circ}$, K. Furnival ae ${ }^{\text {, H. Furstenau }}{ }^{\ell}$, J. Fuster ${ }^{\text {i }}$, G. Galeazzi ag, D. Gamba ${ }^{\text {ac }}$, C. Garcia ${ }^{\text {g }}$, J. Garcia ${ }^{\text {aj }}$, C. Gaspar ${ }^{\mathrm{i}}$, U. Gasparini ${ }^{\text {ag }}$, P. Gavillet ${ }^{\text {i }}$, E.N. Gazis ${ }^{\text {ap }, ~ J .-P . ~ G e r b e r ~}{ }^{\text {aa }}$, P. Giacomelli ${ }^{\text {, }}$, K.-W. Glitza v , R. Gokieli i, V.M. Golovatyuk ${ }^{\text {, }}$, J.J. Gomez Y Cadenas ', A. Goobar ${ }^{\text {m }}$, G. Gopal ${ }^{\text {d }}$, M. Gorski ${ }^{\text {ao }}$, V. Gracco ${ }^{\text {w }}$, A. Grant ${ }^{\mathrm{i}}$,

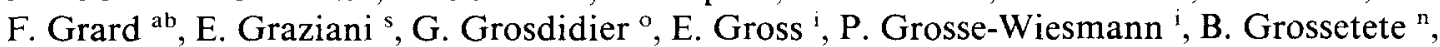
J. Guy ${ }^{\text {d }, ~ F . ~ H a h n ~}{ }^{\text {i }}$, M. Hahn ${ }^{\ell}$, S. Haider ${ }^{\text {p }}$, Z. Hajduk ${ }^{\text {aq }}$, A. Hakansson ${ }^{\text {e }}$, A. Hallgren ${ }^{\text {af }}$, K. Hamacher v, G. Hamel De Monchenault ' , F.J. Harris ', B.W. Heck i, T. Henkes ', I. Herbst ", J.J. Hernandez ${ }^{\mathrm{g}}$, P. Herquet ${ }^{\text {ab }}$, H. Herr ${ }^{\text {i }}$, I. Hietanen ${ }^{\text {ah }}$, C.O. Higgins ${ }^{\text {ae }}$, E. Higon ${ }^{g}$, H.J. Hilke ${ }^{\text {i }}$, S.D. Hodgson ${ }^{t}$, T. Hofmokl ${ }^{\text {ao }}$, R. Holmes ${ }^{a}{ }^{a}$, S.-O. Holmgren ${ }^{m}$, D. Holthuizen ${ }^{p}$, P.F. Honore ${ }^{\text {x }}$ J.E. Hooper ${ }^{\text {an }}$, M. Houlden ${ }^{\text {ac }}$, J. Hrubec ${ }^{b}$, P.O. Hulth ${ }^{m}$, K. Hultqvist ${ }^{m}$, D. Husson ${ }^{\text {aa }}$, P. Ioannou ${ }^{\text {j}}$, D. Isenhower ${ }^{\text {i }}$, P.-S. Iversen ${ }^{\text {h }}$, J.N. Jackson ${ }^{\text {ac }}$, P. Jalocha ${ }^{\text {aq }}$, G. Jarlskog ${ }^{\text {c }}$, P. Jarry ${ }^{\text {c }}$, B. Jean-Marie ${ }^{\text {o }}$, E.K. Johansson ${ }^{m}$, D. Johnson ${ }^{\text {ae }}$, M. Jonker ${ }^{\text {i, }}$ 
L. Jonsson ${ }^{\text {e }}$, P. Juillot ${ }^{\text {aa }}$, G. Kalkanis ${ }^{j}$, G. Kalmus ${ }^{\text {d }}$, F. Kapusta ${ }^{\text {n }}$, S. Katsanevas ${ }^{j}$, E.C. Katsoufis ${ }^{\text {ap }}$, R. Keranen ${ }^{\text {ah }}$, J. Kesteman ${ }^{\text {ab }}$, B.A. Khomenko ${ }^{\text {f }}$, N.N. Khovanski ${ }^{\text {f }}$, B. King ${ }^{\text {ae }}$, N.J. Kjaer ${ }^{\text {i }}$, H. Klein ${ }^{\text {i }}$, W. Klempt ${ }^{\text {i }}$, A. Klovning ${ }^{\text {}}$, P. Kluit ${ }^{\text {p }}$, A. Koch-Mehrin ${ }^{\text {v }}$, J.H. Koehne ${ }^{\ell}$, B. Koene ${ }^{\text {p }}$, P. Kokkinias ${ }^{z}$, M. Kopf ${ }^{\ell}$, M. Koratzinos ${ }^{\text {ac }}$, K. Korcyl ${ }^{\text {aq }}$, A.V. Korytov ${ }^{\text {f }}$, V. Kostiukhin ${ }^{y}$, C. Kourkoumelis ${ }^{j}$, T. Kreuzberger ${ }^{\text {b }}$, J. Krolikowski ${ }^{\text {ao }}$, I. Kronkvist ${ }^{\text {e }}$, J. Krstic ${ }^{\text {t }}$ U. Kruener-Marquis ${ }^{\text {v }}$, W. Krupinski aq, W. Kucewicz ${ }^{\text {, }}$, K. Kurvinen ${ }^{\text {ah }}$, C. Lacasta ${ }^{\text {g }}$, C. Lambropoulos ${ }^{z}$, J.W. Lamsa ${ }^{\text {a }}$, L. Lanceri ${ }^{r}$, V. Lapin ${ }^{y}$, J.-P. Laugier ${ }^{c}$, R. Lauhakangas ${ }^{\text {ah }}$, G. Leder ${ }^{b}$, F. Ledroit ${ }^{\mathrm{a}}$, R. Leitner ${ }^{\mathrm{i}}$, Y. Lemoigne ${ }^{\text {c }}$, J. Lemonne ${ }^{\text {ab }}$, G. Lenzen ${ }^{\text {v }}$ V. Lepeltier ${ }^{\circ}$, A. Letessier-Selvon ${ }^{n}$, E. Lieb ${ }^{\text {v }, ~ D . ~ L i k o ~}{ }^{b}$, E. Lillethun ${ }^{\text {h }}$, J. Lindgren ${ }^{\text {ah }}$, A. Lipniacka ${ }^{\text {ao }}$, I. Lippi ${ }^{\text {ag }}$, R. Llosa ${ }^{\text {am }}$, B. Loerstad ${ }^{\text {e }}$, M. Lokajicek ${ }^{\mathrm{f}}$, J.G. Loken ${ }^{\mathrm{t}}$, A. Lopez-Fernandez ${ }^{\circ}$, M.A. Lopez Aguera ${ }^{\mathrm{aj}}$, M. Los ${ }^{\mathrm{p}}$, D. Loukas ${ }^{\text {, }}$ A. Lounis ${ }^{\text {aa }}$, J.J. Lozano ${ }^{\text {g }}$, P. Lutz ${ }^{\text {x }}$, L. Lyons ${ }^{\text {t }}$, G. Maehlum ${ }^{\text {i }}$, N. Magnussen ${ }^{\text {v }}$, J. Maillard x, A. Maltezos ${ }^{\text {z }}$, F. Mandl ${ }^{\text {b }}$, J. Marco aj, M. Margoni ag, J.-C. Marin i, A. Markou ${ }^{\text {z, }}$

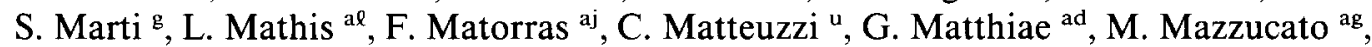
M. McCubbin ae, R. McKay ${ }^{\text {a }}$, R. McNulty ${ }^{\text {ae }}$, E. Menichetti ${ }^{\text {ac }}$, G. Meola ${ }^{\text {w }}$, C. Meroni ${ }^{u}$, W.T. Meyer ${ }^{\text {al }}$, M. Michelotto ${ }^{\text {ag }}$, W.A. Mitaroff ${ }^{b}$, G.V. Mitselmakher ${ }^{f}$, U. Mjoernmark ${ }^{e}$, T. Moa ${ }^{\text {m}}$, R. Moeller ${ }^{\text {an }}$, K. Moenig ${ }^{\text {i, M.R. Monge }}{ }^{\text {w }}$, P. Morettini ${ }^{\text {w }}$, H. Mueller ${ }^{\ell}$, W.J. Murray ${ }^{\text {d }}$, B. Muryn ${ }^{\circ}$, G. Myatt ${ }^{t}$, F. Naraghi ${ }^{n}$, U. Nau-Korzen ${ }^{\text {v }}$, F.L. Navarria ${ }^{\text {ak }}$, P. Negri ${ }^{\text {u }}$, B.S. Nielsen ${ }^{\text {an }}$, B. Nijjhar ${ }^{\text {ae }}$, V. Nikolaenko ${ }^{y}$, V. Obraztsov ${ }^{y}$, K. Oesterberg ${ }^{\text {ah }}$, A.G. Olshevski ${ }^{\mathrm{f}}$, R. Orava ${ }^{\text {ah }}$, A. Ostankov ${ }^{\mathrm{y}}$, A. Ouraou ${ }^{\mathrm{c}}$, M. Paganoni ${ }^{\mathrm{u}}$, R. Pain ${ }^{\mathrm{n}}$, H. Palka ${ }^{\mathrm{p}}$, T. Papadopoulou ${ }^{\text {ap }}$, L. Pape ${ }^{\mathrm{i}}$, A. Passeri s ${ }^{\mathrm{s}}$ M. Pegoraro ${ }^{\text {ag }}$, J. Pennanen ${ }^{\text {ah }}$, V. Perevozchikov ${ }^{\mathrm{y}}$, M. Pernicka ${ }^{\text {b }}$, A. Perrotta ${ }^{\text {ak }}$, F. Pierre ${ }^{c}$, M. Pimenta ${ }^{\text {a }}$, O. Pingot ${ }^{\text {ab }}$, M.E. Pol ${ }^{i}$, G. Polok ${ }^{\text {aq }}$, P. Poropat ${ }^{r}$, P. Privitera ${ }^{\ell}$, A. Pullia ${ }^{u}$, D. Radojicic ${ }^{\natural}$, S. Ragazzi ${ }^{\text {u, P.N. Ratoff }}{ }^{\text {ar }}$, A.L. Read ${ }^{\text {ai }}$, N.G. Redaelli " , M. Regler ${ }^{\text {b }}$, D. Reid ${ }^{\text {ae }}$, P.B. Renton ${ }^{\mathrm{t}}$, L.K. Resvanis ${ }^{\mathrm{j}}$, F. Richard ${ }^{\circ}$, M. Richardson ${ }^{\text {ac }}$, J. Ridky ${ }^{\text {f }}$, G. Rinaudo ${ }^{\text {ac }}$, I. Roditi i , A. Romero ${ }^{\text {ac }}$, I. Roncagliolo ${ }^{\text {w }}$, P. Ronchese ${ }^{\text {ag }}$, C. Ronnqvist ${ }^{\text {ah }}$, E.I. Rosenberg al, U. Rossi ${ }^{\text {ak }}$, E. Rosso ${ }^{i}$, P. Roudeau ${ }^{\circ}$, T. Rovelli ${ }^{\text {ak }}$, W. Ruckstuhl ${ }^{\text {}}$, V. Ruhlmann ${ }^{c}$, A. Ruiz ${ }^{\text {aj }}$, K. Rybicki ${ }^{\text {aq }}$, H. Saarikko ${ }^{\text {ah }}$, Y. Sacquin ${ }^{\text {c }}$, G. Sajot ${ }^{\text {q }}$, J. Salt ${ }^{\mathrm{g}}$, E. Sanchez ${ }^{\mathrm{g}}$, J. Sanchez ${ }^{\mathrm{am}}$, M. Sannino ${ }^{\text {w }}$, M. Schaeffer ${ }^{\text {aa }}$, S. Schael ${ }^{\ell}$, H. Schneider ${ }^{\ell}$, M.A.E. Schyns ${ }^{i}$, F. Scuri ${ }^{~}$, A.M. Segar ${ }^{t}$, R. Sekulin ${ }^{d}$, M. Sessa ${ }^{r}$, G. Sette ${ }^{\text {w }}$, R. Seufert ${ }^{\ell}$, R.C. Shellard ${ }^{i}$, P. Siegrist ${ }^{c}$, S. Simonetti ${ }^{\text {w }}$, F. Simonetto ${ }^{\text {ag, }}$, A.N. Sissakian ${ }^{\text {f }}$, T.B. Skaali ai , G. Skjevling ${ }^{a i}$, G. Smadja ${ }^{c, k}$, N. Smirnov ${ }^{y}$, G.R. Smith ${ }^{\text {d }}$, R. Sosnowski ao, T.S. Spassoff q , E. Spiriti s, S. Squarcia ${ }^{w}$, H. Staeck ${ }^{\text {v }}$, C. Stanescu s, G. Stavropoulos ${ }^{\text {z }}$, F. Stichelbaut ${ }^{\text {ab }}$, A. Stocchi ${ }^{\text {, }}$ J. Strauss ${ }^{\text {b }}$, R. Strub ${ }^{\text {aa }}$, M. Szczekowski ${ }^{\text {ao }}$, M. Szeptycka ${ }^{\text {ao }}$, P. Szymanski ${ }^{\text {ao }}$, T. Tabarelli ${ }^{\text {u}}$, S. Tavernier ${ }^{\text {ab }}$, O. Tchikilev ${ }^{y}$, G.E. Theodosiou ${ }^{z}$, A. Tilquin ${ }^{\text {as }}$, J. Timmermans ${ }^{p}$, V.G. Timofeev ${ }^{f}$, L.G. Tkatchev ${ }^{f}$, T. Todorov $^{f}$, D.Z. Toet ${ }^{\mathrm{p}}$, O. Toker ${ }^{\text {ah }}$, E. Torassa ${ }^{\text {ac }}$, L. Tortora $^{\mathrm{s}}$, M.T. Trainor ${ }^{\mathrm{t}}$, D. Treille ${ }^{\mathrm{i}}$, U. Trevisan ${ }^{w}$, W. Trischuk ${ }^{i}$, G. Tristram ${ }^{x}$, C. Troncon ${ }^{\text {, }}$, A. Tsirou ${ }^{i}$, E.N. Tsyganov ${ }^{f}$, M. Turala aq, R. Turchetta ${ }^{\text {aa }}$, M.-L. Turluer ${ }^{c}$, T. Tuuva ah , I.A. Tyapkin ${ }^{\text {f }}$, M. Tyndel ${ }^{d}$, S. Tzamarias ${ }^{i}$, B. Ueberschaer ${ }^{v}$, S. Ueberschaer ${ }^{v}$, O. Ullaland ${ }^{i}$, V. Uvarov ${ }^{y}$, G. Valenti ${ }^{\text {ak }}$, E. Vallazza ${ }^{\text {ac }}$, J.A. Valls Ferrer ${ }^{\mathrm{g}}$, C. Van der Velde ${ }^{\mathrm{ab}}$, G.W. Van Apeldoorn ${ }^{\mathrm{p}}$, P. Van Dam ${ }^{\mathrm{p}}$, W.K. Van Doninck ${ }^{\text {ab }}$, J. Varela ${ }^{\text {a }}$, P. Vaz ${ }^{i}$, G. Vegni ${ }^{\text {u }}$, L. Ventura ${ }^{\text {ag }}$, W. Venus ${ }^{d}$, F. Verbeure ${ }^{\text {ab }}$, L.S. Vertogradov ${ }^{f}$, L. Vibert ${ }^{n}$, D. Vilanova ${ }^{c}$, L. Vitale ${ }^{r}$, E. Vlasov ${ }^{y}$, E.V. Vlasov ${ }^{y}$,

S. Vlassopoulos ${ }^{a p}$, A.S. Vodopyanov ${ }^{f}$, M. Vollmer ${ }^{\mathrm{v}}$, S. Volponi ${ }^{\text {ak }}$, G. Voulgaris ${ }^{j}$, M. Voutilainen ${ }^{\text {ah }}$, V. Vrba ${ }^{\mathrm{s}}$, H. Wahlen ${ }^{\mathrm{v}}$, C. Walck ${ }^{\mathrm{m}}$, F. Waldner ${ }^{\mathrm{r}}$, M. Wayne ${ }^{\mathrm{a} \ell}$, P. Weilhammer ${ }^{\text {i }}$, J. Werner ${ }^{\text {v }}$, A.M. Wetherell ${ }^{\text {, }}$ J.H. Wickens ${ }^{\text {ab }}$, J. Wikne ${ }^{\text {ai }}$, G.R. Wilkinson ${ }^{\text {t }}$ W.S.C. Williams ${ }^{\mathrm{t}}$, M. Winter ${ }^{\text {aa }}$, D. Wormald ${ }^{\text {ai }}$, G. Wormser ${ }^{\circ}$, K. Woschnagg ${ }^{\text {af }}$, N. Yamdagni ${ }^{\mathrm{m}}$, P. Yepes ${ }^{\mathrm{i}}$, A. Zaitsev ${ }^{\mathrm{y}}$, A. Zalewska ${ }^{\text {aq }}$, P. Zalewski ${ }^{\circ}$, D. Zavrtanik ${ }^{\mathrm{i}}$, 
E. Zevgolatakos ${ }^{\text {, }}$, G. Zhang ${ }^{\mathrm{v}}$, N.I. Zimin ${ }^{\mathrm{f}}$, M. Zito ${ }^{\mathrm{c}}$, R. Zitoun ${ }^{\mathrm{n}}$, R. Zukanovich Funchal ${ }^{\mathrm{x}}$, G. Zumerle ${ }^{\mathrm{ag}}$ and J. Zuniga ${ }^{\mathrm{g}}$

a LIP, Av. Elias Garcia 14 - 1e, P-1000 Lisbon Codex, Portugal

b Institut für Hochenergiephysik, Österreichische Akademie der Wissenschaften, Nikolsdorfergasse 18, A-1050 Vienna, Austria

c DPhPE, CEN-Saclay, F-91191 Gif-Sur-Yvette Cedex, France

d Rutherford Appleton Laboratory, Chilton, Didcot OX11 $0 Q X, U K$

- Department of Physics, University of Lund, Sölvegatan 14, S-22363 Lund, Sweden

f Joint Institute for Nuclear Research, Dubna, Head Post Office, P.O. Box 79, SU-101000 Moscow, USSR

\& Instituto de Fisica Corpuscular (IFIC), Centro Mixto Universidad de Valencia-CSIC, and Departamento de Fisica Atomica, Molecular y Nuclear, Universidad de Valencia, Avda. Dr. Moliner 50, E-46100 Burjassot (Valencia), Spain

" Department of Physics, University of Bergen, Allégaten 55, N-5007 Bergen, Norway

i CERN, CH-1211 Geneva 23, Switzerland

j Physics Laboratory, University of Athens, Solonos Street 104, GR-10680 Athens, Greece

* Université Claude Bernard de Lyon, 43 Boulevard du 11 Novembre 1918, F-69622 Villeurbanne Cedex, France

${ }^{\natural}$ Institut für Experimentelle Kernphysik, Universität Karlsruhe, Pf. 6980, W-7500 Karlsruhe 1, FRG

m Institute of Physics, University of Stockholm, Vanadisvägen 9, S-113 46 Stockholm, Sweden

" LPNHE, Universités Paris VI et VII, Tour 33 (RdC), 4 place Jussieu, F-75230 Paris Cedex 05, France

- Laboratoire de l'Accélérateur Linéaire, Université de Paris-Sud, Bâtiment 200, F-91405 Orsay, France

p NIKHEF-H, Postbus 41882, NL-1009 DB Amsterdam, The Netherlands

ч Institut des Sciences Nucléaires, Université de Grenoble 1, F-38026 Grenoble, France

r Dipartimento di Fisica, Università di Trieste and INFN, Via A. Valerio 2, I-34127 Trieste, Italy and Istituto di Fisica, Università di Udine, Via Larga 36, I-33100 Udine, Italy

s Istituto Superiore di Sanità. Istituto Nazionale di Fisica Nucleare (INFN), Viale Regina Elena 299, I-00161 Rome, Italy

' Nuclear Physics Laboratory, University of Oxford, Keble Road, Oxford OXI 3RH, UK

" Dipartimento di Fisica, Università di Milano and INFN, Via Celoria 16, I-20133 Milan. Italy

" Fachbereich Physik, Universität Wuppertal, Pf. 100 127, W-5600 Wuppertal 1, FRG

" Dipartimento di Fisica, Università di Genova and INFN, Via Dodecaneso 33, 1-16146 Genoa, Italy

* Laboratoire de Physique Corpusculaire, College de France, 11 place M. Berthelot, F-75231 Paris Cedex 5, France

y Institute for High Energy Physics, Serpukhov, P.O. Box 35, SU-142284 Protvino (Moscow Region), USSR

2 Institute of Nuclear Physics, N.C.S.R. Demokritos, P.O. Box 60228, GR-15310 Athens, Greece

aa Division des Hautes Energies, CRN-Groupe DELPHI and LEPSI, B.P. 20 CRO, F-67037 Strasbourg Cedex, France

ab Physics Department, Universitaire Instelling Antwerpen, Universiteitsplein 1, B-2610 Wilrijk, Belgium and IIHE, ULB-VUB, Pleinlaan 2, B-1050 Brussels, Belgium and Service de Physique des Particules Elémentaires, Faculté des Sciences, Université de l'Etat Mons, Av. Maistriau 19, B-7000 Mons, Belgium

ac Dipartimento di Fisica Sperimentale, Università di Torino and INFN, Via P. Giuria I, I-10125 Turin, Italy

ad Dipartimento di Fisica, Università di Roma II and INFN, Tor Vergata, I-00173 Rome, Italy

ae Department of Physics, University of Liverpool, P.O. Box 147, Liverpool L69 3BX,UK

af Department of Radiation Sciences, University of Uppsala, P.O. Box 535, S-75121 Uppsala, Sweden

ag Dipartimento di Fisica, Università di Padova and INFN, Via Marzolo 8, 1-35131 Padua, Italy

ah Department of High Energy Physics, University of Helsinki, Siltavuorenpenger $20 \mathrm{C}, \mathrm{SF}-00170 \mathrm{Helsinki} 17$, Finland

ai Physics Depariment, University of Oslo, Blindern, N-1000 Oslo 3, Norway

aj Facultad de Ciencias, Universidad de Santander, av. de los Castros, E-39005 Santander, Spain

${ }^{\text {ak }}$ Dipartimento di Fisica, Università di Bologna and INFN, Via Irnerio 46, I-40126 Bologna, Italy

al Ames Laboratory and Department of Physics, Iowa State University, Ames, IA 50011, USA

am Universidad Complutense, Avda. Complutense $s / n$, E-28040 Madrid, Spain

an Niels Bohr Institute, Blegdamsvej 17, DK-2100 Copenhagen $\emptyset$, Denmark

ao Institute for Nuclear Studies, and University of Warsaw, Ul. Hoza 69, PL-0068I Warsaw, Poland

ap Physics Department, National Technical University, Zografou Campus, GR-15773 Athens, Greece

aq High Energy Physics Laboratory, Institute of Nuclear Physics, Ul. Kawiory 26 a, PL-30055 Cracow 30, Poland

ar School of Physics and Materials, University of Lancaster, Lancaster LAI $4 Y B, U K$

as Université d'Aix-Marseille II, Case 907, 70, route Léon Lachamp, F-13288 Marseille Cedex 09, France

Received 30 October 1991 


\begin{abstract}
We have searched for possible fourth family heavy neutrinos, pair produced in $Z^{0}$ decays, in a sample of about 112000 hadronic $Z^{0}$ final states collected with the DELPHI detector. For all mixing matrix elements we exclude a new Dirac neutrino lighter than $44.5 \mathrm{GeV}$ at a $95 \%$ confidence level, if the neutrino couples to the electron or muon family, and lighter than $44.0 \mathrm{GeV}$, if the neutrino couples to the tau family. Depending on the values of the mixing element and to which lepton family the neutrino couples, we obtain mass limits up to $46.2 \mathrm{GeV}$. For all mixing matrix elements we exclude a new Majorana neutrino lighter than 39.0 GeV, if it couples to the electron or the muon family, and lighter than $38.2 \mathrm{GeV}$, if it couples to the tau family. Depending on the values of the mixing matrix element and to which lepton family the neutrino couples, we obtain mass limits up to 44.7 $\mathrm{GeV}$. We also exclude stable new Dirac neutrinos lighter than $45.0 \mathrm{GeV}$ and new Majorana neutrinos lighter than $39.5 \mathrm{GeV}$.
\end{abstract}

\section{Introduction}

Heavy neutral leptons that have not so far been observed are predicted by various theories [1]. A sequential fourth generation neutrino is the most natural extension. From indirect measurements of the invisible width, $\Gamma_{\text {inv }}$, of the $Z^{0}$-resonance the number of light neutrinos can be estimated. A combination of the results from the LEP experiments [2] gives $N_{\nu}=2.99 \pm 0.05$ (the width associated with a massless neutrino in the standard model is taken to be $166.5 \pm 0.5 \mathrm{MeV}$ ). This assumes however that the lifetime of the neutrino is long enough for it to escape the apparatus before decaying. If the neutrino has a shorter lifetime a direct search is the best way to look for it.

The LEP collider at CERN provides opportunity to search for heavy neutrinos because of the large cross sections at the $Z^{0}$ resonance. Here we present results of a direct search for neutrinos with mass up to the half of the $Z^{0}$ mass, using data taken with the DELPHI detector. We also use $\Gamma_{\text {inv }}$ to exclude long-lived neutrinos and $\Gamma_{\text {tot }}$ to exclude neutrinos independently of the mixing matrix element. It has been proposed that right-handed neutrinos mix with the known neutrinos and reduce the measurable invisible width [3] but this possibility is not taken into account here. We restrict the analysis to massive neutrinos, $L^{0}$, belonging to a new electroweak doublet $\left(\begin{array}{c}L \\ L^{0}\end{array}\right)$, which mixes with the three known lepton doublets, just as the quark doublets mix in the standard model.

We consider both the case in which the neutrino is a Dirac particle and the case in which it is a Majorana particle. The neutrino, $L^{0}$, is in general a superposition of all neutrino mass eigenstates:

$L^{0}=\sum_{l} U_{l L} \nu_{l}^{\prime}$ where $\nu_{l}^{\prime}$ is $\nu_{e}^{\prime}, \nu_{\mu}^{\prime}, \nu_{\tau}^{\prime}$ or $L^{0 \prime}$ and $U_{I L}$ is the mixing matrix. The neutrinos can be pair produced in LEP via the $Z^{0}$ boson and decay through a flavour changing charged current reaction

$L^{0} \rightarrow l^{-} W^{+*}$.

Flavour changing neutral currents are forbidden by the GIM-mechanism [4], and are not considered here. The lifetime of the Dirac neutrino in terms of its leptonic branching ratio and the muon lifetime, $\tau_{\mu}$, is given by

$\tau_{L^{0}}=\left(\frac{m_{\mu}}{m_{L^{0}}}\right)^{5} \frac{\tau_{\mu} \sum_{l \neq L} \operatorname{Br}\left(L^{0} \rightarrow l^{-} e^{+} \nu_{e}\right)}{f \sum_{l \neq L}\left|U_{l L}\right|^{2}}$,

where $f$ is a phase-space suppression factor for final states particles, which differs appreciably from unity when the mass difference between the neutrino and its decay products is small [5]. The lifetime of a Majorana neutrino is half of the lifetime for the corresponding Dirac neutrino, since the decays $L^{0} \rightarrow l^{-} W^{+*}$ and $L^{0} \rightarrow l^{+} W^{-*}$ occur with equal probability for a Majorana neutrino. The phase-space factor $f$ is assumed to be equal to unity in the whole mass range considered in the direct search. The expected cross section for the pair production is

$\sigma_{L^{0} \bar{L}^{0}}=\sigma_{\nu \bar{\nu}} T(\beta)$,

where

$T(\beta)=\beta^{3} \quad($ Majorana $)$,

$T(\beta)=\frac{1}{4} \beta\left(3+\beta^{2}\right) \quad($ Dirac $)$,

assuming the same couplings to the $Z^{0}$ as for the light neutrinos. $\sigma_{\nu \nu}$ is the cross section for pair production of a massless neutrino and $\beta\left(=\sqrt{1-4 m_{L 0}^{2} / s}\right)$ is the velocity of the produced particle. The branching ratios for the different decay channels of a pair of heavy neutrinos are given in table 1 , assuming that the doublet mixes with only one of the known lepton doublets $(l=e, \mu$ or $\tau)$. 
Table 1

The different decay channels of the heavy neutrino, their branching ratios and relative cross section compared with the total hadronic cross section (observe the kinematic factor $T(\beta)$ of eq. (5) in the expression for $R$ ).

\begin{tabular}{lll}
\hline Decay channel & $\operatorname{Br}\left(L^{0} \bar{L}^{0} \rightarrow\right.$ channel $)$ & $R / T(\beta)=\Gamma_{\nu} \operatorname{Br}\left(L^{0} \bar{L}^{0} \rightarrow\right.$ channel $) / \Gamma_{\mathrm{h}}$ \\
\hline$L^{0} \bar{L}^{0} \rightarrow l l q \bar{q}^{\prime} q \bar{q}^{\prime}$ & 0.461 & 0.0440 \\
$L^{0} \bar{L}^{0} \rightarrow l q q \bar{q}^{\prime} l^{\prime} \nu^{\prime}$ & 0.436 & 0.0416 \\
$L^{0} \bar{L}^{0} \rightarrow l l l^{\prime} \nu^{\prime} l^{\prime} \nu^{\prime}$ & 0.103 & 0.0098 \\
all channels & 1.000 & 0.0955 \\
\hline
\end{tabular}

\section{The detector}

The DELPHI detector is described in detail in ref. [6]. Here we summarize the main features of the tracking detectors used in this analysis. Charged tracks are measured in the solenoidal 1.2 T magnetic field with three cylindrical detectors. The Inner Detector (ID) covers polar angles from the beam axis between $29^{\circ}$ and $151^{\circ}$ (with an inner radius $\left(r_{\text {in }}\right)$ of $12 \mathrm{~cm}$ and an outer radius $\left(r_{\text {out }}\right)$ of $28 \mathrm{~cm}$ ), the Time Projection Chamber (TPC) covers angles between $21^{\circ}$ and $159^{\circ}\left(r_{\text {in }}=30 \mathrm{~cm}, r_{\text {out }}=122 \mathrm{~cm}\right)$ and the Outer Detector (OD) covers angles between $42^{\circ}$ and $138^{\circ}\left(r_{\text {in }}=198 \mathrm{~cm}, r_{\text {out }}=206 \mathrm{~cm}\right)$.

The trigger is based on the tracking detectors, the electromagnetic calorimeters and a layer of scintillation counters situated inside the magnet coil in the barrel region and between the two layers of the muon chambers in the forward region.

Detailed information on the trigger can be found in refs. $[6,7]$. The trigger efficiency for a hadronic $Z^{0}$ event with a polar event axis between $20^{\circ}$ and $160^{\circ}$ is $\geqslant 99.7 \%[7]$.

\section{Analysis using widths}

The minimal standard model (MSM) prediction for the total width of the $Z^{0}$-resonance is $\Gamma_{\mathrm{tot}}^{\mathrm{SM}}=$ $2484 \pm 11 \mathrm{MeV}^{\# 1}$, if $\alpha_{\mathrm{s}}$ is taken to be $0.110 \pm 0.006$ [9], the mass of the Higgs to be between $50 \mathrm{GeV}$ and

\#1 The value of the total width was calculated using the formulae of ref. [8]. The error is due to the uncertainty in the top mass and the Higgs mass and the experimental errors in the measurement of $\alpha_{\mathrm{s}}$ and the $Z^{0}$ mass.
$1 \mathrm{TeV}$, the top mass $=139 \pm 38 \mathrm{GeV}[10]$ and the $Z^{0}$ mass $=91.177 \pm 0.0022 \mathrm{GeV}[7]$. The data taken by DELPHI give $\Gamma_{\text {tot }}^{\exp }=2465 \pm 19 \pm 5 \mathrm{MeV}$ [7]. From this, we can estimate the largest partial width which can exist beyond the total width of the known quarks and leptons. Using a gaussian distribution, renormalized to the physical region, we calculate an upper limit for the new partial width so that the area between the bound (here the MSM prediction) and the limit is $95 \%$ of the physical region [11]. We then obtain $\Gamma_{L^{0}}<33 \mathrm{MeV}=0.20 \Gamma_{\nu}$ at a $95 \%$ confidence level, and can thereby exclude all neutrinos with a mass less than $44.0 \mathrm{GeV}$ in the Dirac case and $36.9 \mathrm{GeV}$ in the Majorana case. This is independent of the mixing matrix element and the $33 \mathrm{MeV}$ limit for the width is valid for all new particles.

Secondly by assuming $\Gamma_{\mathrm{inv}}^{\mathrm{SM}}=500 \pm 2 \mathrm{MeV}[7]$ (the MSM prediction of the width of three massless neutrinos), and combining this with a measurement of the invisible width of the $Z^{0}$-resonance using DELPHI data, $\Gamma_{\text {inv }}^{\exp }=488 \pm 7 \pm 12 \mathrm{MeV}[7]$. We can estimate the largest invisible width there can exist beyond the known three neutrinos using the approach above and conclude that $\Gamma_{L^{0}}<21 \mathrm{MeV}=0.12 \Gamma_{v}$.

If the $L^{0}$ events do not contribute to either $\Gamma_{\text {had }}$ or to any $\Gamma_{l}{ }^{\# 2}$ then they must contribute to the $\Gamma_{\text {inv }}$. The probability, $\epsilon_{\text {inv }}$, that an $L^{0}$-event would not be selected by our hadronic $Z^{0}$ event criteria give below, and hence contributes to the $\Gamma_{\mathrm{inv}}$ is

$\epsilon_{\mathrm{inv}}=1-\frac{\epsilon_{L^{0}}(r)}{\epsilon_{\mathrm{h}}}$,

where $\epsilon_{\mathrm{h}}$ is the efficiency to select a hadronic $Z^{0}$ event (in DELPHI $(93.5 \pm 1.0) \%$ [12]) and $\epsilon_{L^{\circ}}$ the efficiency to select a heavy neutrino event as a hadronic $Z^{0}$ event, which is a function of the mean decay length, 


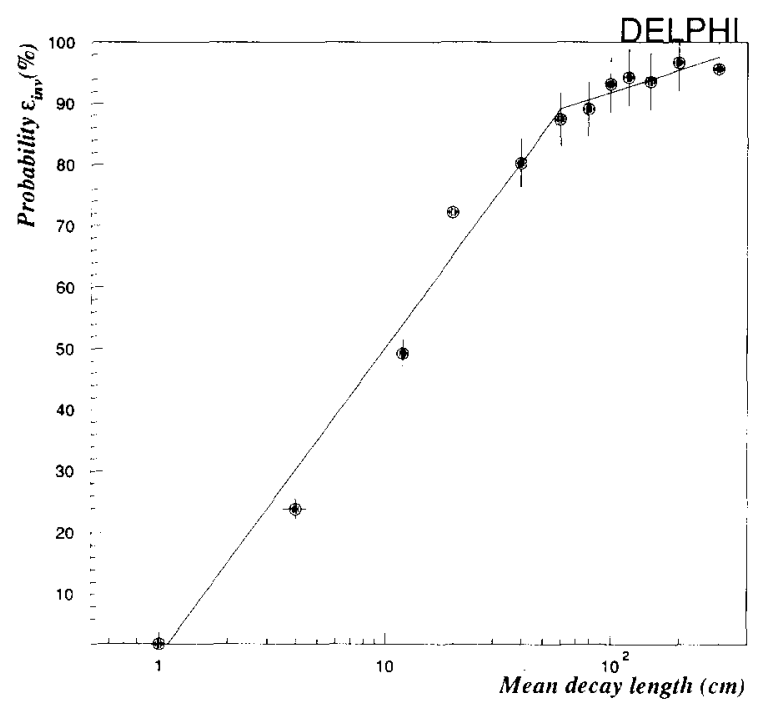

Fig. 1. The probability that a heavy neutrino event contributes to the $\Gamma_{\text {inv }}$ as a function of the logarithm of the mean decay length of the neutrino.

$r$, of the neutrino. This probability was studied with DELSIM, the DELPHI full detector simulation and the result is shown in fig. 1. For a heavy neutrino with a mean decay length of more than $400 \mathrm{~cm}$ the probability is $\approx 100 \%$. In ref. [7] slightly different selection criteria were used for hadronic $Z^{0}$ events than in this analysis, but the resulting differences were smaller than the errors of fig. 1.

We exclude, with $95 \%$ confidence, neutrinos with a mass less than $45.0 \mathrm{GeV}$ in the Dirac case and 39.5 $\mathrm{GeV}$ in the Majorana case with a decay length more than $400 \mathrm{~cm}$. We also exclude smaller mean decay lengths than $400 \mathrm{~cm}$ for neutrinos with smaller masses using the information of the fit in fig. 1 . The results are shown as the curves labelled $\Gamma_{\text {inv }}$ and $\Gamma_{\text {tot }}$ in figs. 5 and 6.

\section{Direct search}

The topology used in the direct search for heavy neutrinos, was two isolated charged particles and at

$\# 2$ No $L^{0}$ event contributes to $\Gamma_{\tau}$ because this width was measured using a topology of one charged particle in one hemisphere and $n$ in the other, while $L^{0}$ events are characterized by at least two charged particles in each hemisphere. least two jets in the event. First the event was required to pass the selection for a hadronic $Z^{0}$ according to the following selection criteria [12]. Only charged particles with the following characteristics are used:

- polar angle, $\theta$, between $20^{\circ}$ and $160^{\circ}$;

- momentum between $0.1 \mathrm{GeV} / c$ and $50 \mathrm{GeV} / c$;

- track length above $30 \mathrm{~cm}$;

- projection of the impact parameter in the $x y$-plane below $4 \mathrm{~cm}$;

$-z$ coordinate at the origin below $10 \mathrm{~cm}$;

- relative error on momentum measurement below $100 \%$.

An event is required to have at least 3 charged particles in one hemisphere $\left(\theta>90^{\circ}\right.$ or $\left.\theta<90^{\circ}\right)$ and the sum of the transverse momentum squared of all particles relative to the beam axis must be greater than 9 $\mathrm{GeV}^{2} / c^{2}$. The efficiency for selecting a hadronic $Z^{0}$ event is $93.5 \%$ [12] and a background of about $1.3 \%$ of leptonic $Z^{0}$ final states (tau pairs) and $0.2 \%$ of non$Z^{0}$ events (beam-gas interaction and two photon events) remain after applying these criteria [12].

The isolation of a particle is specified by the isolation parameter $\rho$. First the particle is removed, and the jets are constructed from the remaining particles. A particle is considered to be isolated if the $\rho$ of the particle is bigger than $2.15 \mathrm{GeV}^{1 / 2}$, where $\rho$ is computed from the jet which is closest to the particle track and

$\rho=\sqrt{2 E_{\text {particle }}\left(1-\cos \theta_{\text {particle,jet }}\right)}$.

Furthermore it is required that the angle between the closest jet and the particle track has to be greater than $45^{\circ}\left(\cos \theta_{\text {particle, jet }}<0.71\right)$ and the momentum of the particle greater than $4 \mathrm{GeV} / c$. These cuts were made to optimize the ratio between the signal and the background.

A heavy neutrino candidate should contain two isolated particles and all the other particles should form at least two jets, defined by the Lund cluster algorithm LUCLUS [13], using the default value of the resolution parameter $\left(d_{\text {join }}=2.5 \mathrm{GeV}\right)$.

For a given number of hadronic $Z^{0}$ final states, $N_{\mathrm{h}}$, the corresponding number of heavy neutrino events, $N_{L^{0}}$, is

$N_{L^{0}}=\frac{\epsilon N_{\mathrm{h}} \Gamma_{L^{0}}}{\epsilon_{\mathrm{h}} \Gamma_{\mathrm{h}}+\epsilon_{\mathrm{L}^{0}} \Gamma_{\mathrm{L}^{0}}}$ 
Table 2

The number of the hadronic $Z^{0}$ final states, $N_{\mathrm{h}}$, taken at different centre of mass energies, $\sqrt{s}$.

\begin{tabular}{lr}
\hline$\sqrt{s}(\mathrm{GeV})$ & \multicolumn{1}{c}{$N_{\mathrm{h}}$} \\
\hline 88.223 & 1586 \\
89.224 & 3620 \\
90.222 & 6711 \\
91.225 & 82587 \\
92.220 & 8715 \\
93.221 & 5620 \\
94.218 & 3529 \\
total & 112368 \\
\hline
\end{tabular}

where $\epsilon$ is the total efficiency to select a heavy neutrino event as a heavy neutrino candidate. $\Gamma_{\mathrm{h}}$ is the total width associated with the five known quarks and $\Gamma_{L^{0}}$ the width associated with the heavy neutrino. The real data sample used consisted of 112368 hadronic $Z^{0}$ events collected by DELPHI in 1990 and table 2 shows how they are distributed over the different centre of mass energies. The expected number of heavy neutrino events as a function of the neutrino mass is shown in fig. 2.

The efficiency, $\epsilon$, depends on the mass of the neutrino, $m_{L^{0}}$, and the decay length of the neutrino, $r$ $\left(=\gamma \beta c \tau_{L^{0}}\right)$, as the mass of the neutrino determines the isolation of the lepton produced and the decay

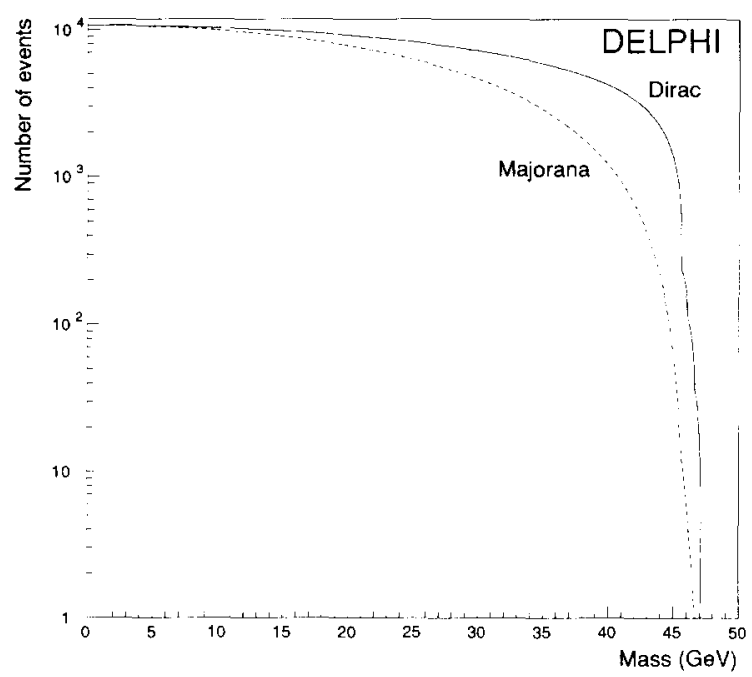

Fig. 2. The number of produced heavy neutrino events as a function of the mass of the neutrino.

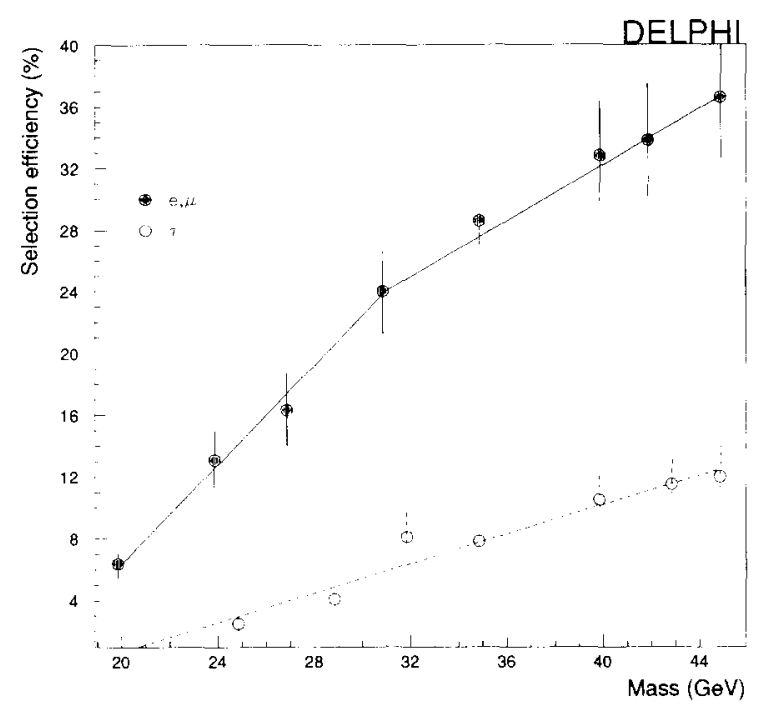

Fig. 3. The selection efficiency for a heavy neutrino event as a function of the mass $(r=0.01 \mathrm{~cm})$.

length determines the efficiency to select the event as a hadronic $Z^{0}$. A study of the selection efficiency with different $m_{L^{0}}$ and $r$ was made, using the DELPHI full detector simulation, and analyzing the Monte Carlo events through the same chain as the real data. The results of this study are shown in figs. 3 and 4.

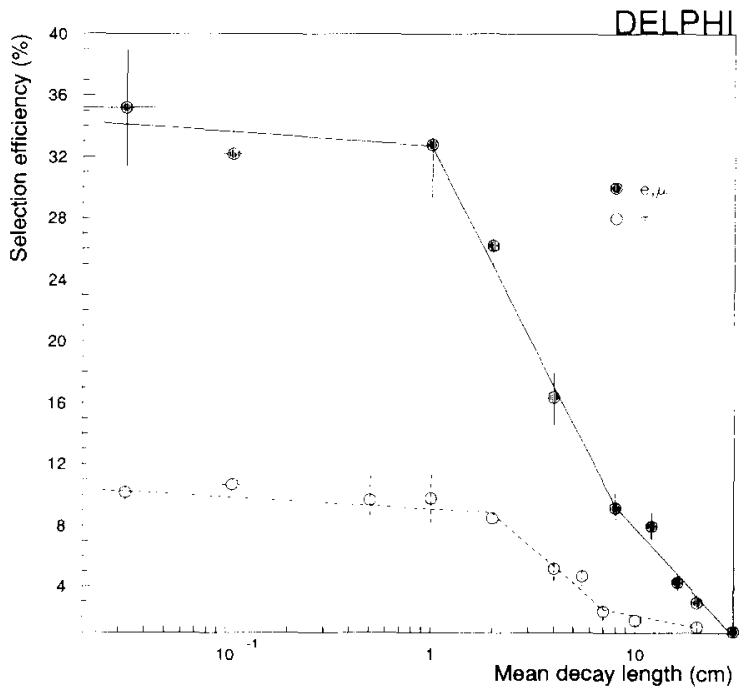

Fig. 4. The selection efficiency for a heavy neutrino event as a function of the logarithm of the mean decay length $\left(m_{L}=40\right.$ $\mathrm{GeV})$. 


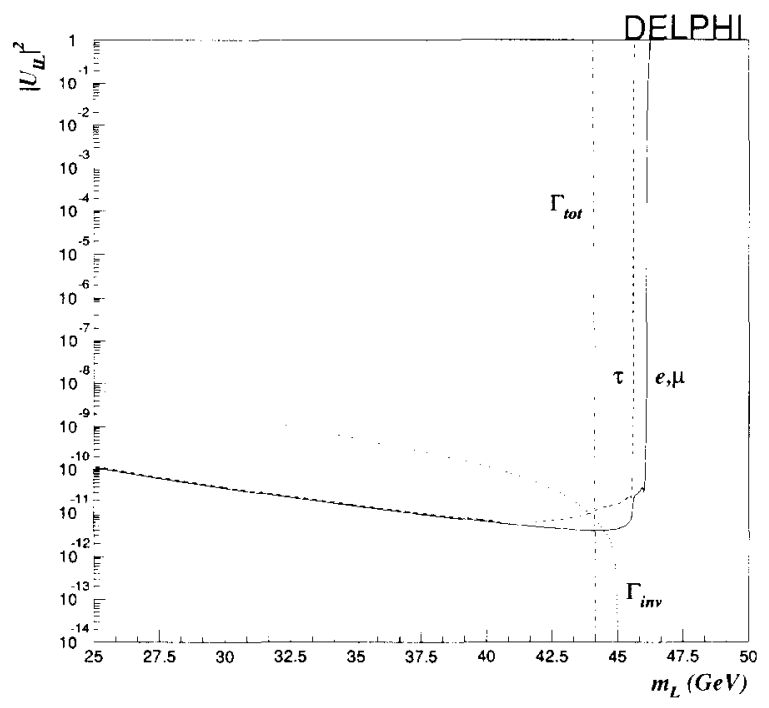

Fig. 5. The $95 \%$ CL limits on $\left|U_{l L}\right|^{2}$ as a function of $m_{L}$ for $e, \mu$ and $\tau$ mixing for a Dirac neutrino, assuming mixing with one family.

We estimate from a sample of simulated $q \bar{q}$ events, comparable in size to the real data, that there is in the real data a background of $78 \pm 10$ events fulfilling the selection criteria.

In the real data sample we found 94 events fulfilling the selection criteria of two isolated particles and at least two jets. This gives a hypothetical signal of $+16 \pm 14$. No peak was found in the reconstruction invariant mass spectrum of the candidates. Using the same method as before, we can exclude all mass regions in which we expect to have a signal of more than 40 events.

From eqs. (4), (5) and (8) we get a constraint for $T(\beta)$, from which the upper mass limits for both Majorana and Dirac neutrinos were determined. We calculate using eq. (8) for each neutrino mass the selection efficiency, $\epsilon$, for which 40 events would pass our selection criteria. The corresponding mean decay length is obtained by using the information of the fits in figs. 3 and 4 . The limits obtained are shown in figs. 5 and 6 as a function of the mixing matrix element squared, $\left|U_{I L^{0}}\right|^{2}$, and $m_{L^{0}}$. They are labelled according to which lepton family the neutrino mixes with.

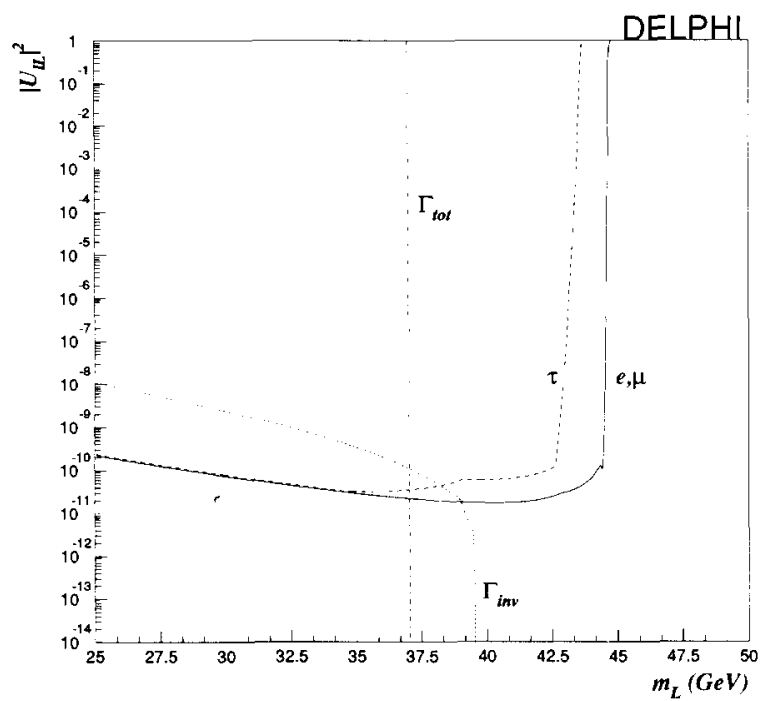

Fig. 6. The $95 \% \mathrm{CL}$ limits on $\left|U_{l L}\right|^{2}$ as a function of $m_{L}$ for $e, \mu$ and $\tau$ mixing for a Majorana neutrino, assuming mixing with one family.

\section{Results and conclusions}

No evidence for the existence of a heavy neutrino was found in the analysis. Dirac neutrinos coupling to the electron and muon families were excluded for all mixing matrix elements with a mass less than 44.5 $\mathrm{GeV}$, and those coupling to the tau with a mass less than $44.0 \mathrm{GeV}$. For Dirac neutrinos also neutrinos with very small mixing matrix elements were excluded up to $45.0 \mathrm{GeV}$, and for mixing matrix elements squared bigger than $10^{-11}$ up to $46.2 \mathrm{GeV}$ for neutrinos coupling to the electron and muon families and up to $45.7 \mathrm{GeV}$ for neutrinos coupling to the tau family. For Majorana neutrinos coupling to the electron and muon families the mass limit is $39.0 \mathrm{GeV}$, and coupling to the tau family the mass limit is 38.2 $\mathrm{GeV}$. Depending on the values of the mixing matrix element and to which lepton family the neutrino couples, we obtain mass limits up to $44.7 \mathrm{GeV}$ for neutrinos coupling to the electron and muon families and up to $43.6 \mathrm{GeV}$ for neutrinos coupling to the tau family. The limits in the case where the new heavy neutrino mixes arbitrarily with all known doublets are the same as if the neutrino couples to the tau family. The regions excluded by this analysis in the mixing matrix element squared versus mass of the neutrino 
plane are shown in figs. 5 (Dirac) and 6 (Majorana). The mass limits given for a Dirac neutrino agree with other LEP results $[14,15]$ and results from SLC [16] and the limit where all mixing matrix elements are excluded is higher in this analysis than in any other. For a Majorana neutrino the limits from the direct search are not as high as those of the L3 Collaboration [15] but the limit where all mixing matrix elements are excluded is considerably higher in this analysis.

\section{Acknowledgement}

We are greatly indebted to our technical collaborators and to the funding agencies for their support in building and operating the DELPHI detector, and to the members of the CERN-SL Division for the superb performance of the LEP collider.

\section{References}

[1] J.C. Pati and A. Salam, Phys. Lett. B 58 (1975) 333; R.N. Mohapatra and G. Senjanovic, Phys. Rev. Lett. 44 (1980) 912;

M. Gell-Mann, P. Ramond and R. Slansky, in: Supergravity, eds. D.Z. Freedman and P. van Nieuwenhuizen (NorthHolland, Amsterdam, 1980) p. 315;

J. Maalampi and K. Enqvist, Phys. Lett. B 97 (1980) 217;
M. Gronau, C.N. Leung and J.L. Rosner, Phys. Rev. D 29 (1984) 2539;

J. Bagger et al., Nucl. Phys. B 258 (1985) 565.

[2] J.R. Carter, Precision tests of the standard model at LEP, invited talk at the LP-HEP Conf. (Geneva, 1991).

[3] C. Jarlskog, Phys. Lett. B 241 (1990) 579.

[4] S.L. Glashow, J. Iliopoulos and L. Maiani, Phys. Rev. D 2 (1970) 1285 .

[5] See e.g. R.E. Shrock, Phys. Rev. D 24 (1981) 1275.

[6] DELPHI Collab., P. Aarnio et al., Nucl. Instrum. Methods A 303 (1991) 233 .

[7] DELPHI Collab., P. Abreu et al,, Nucl. Phys. B 367 (1991) 511.

[8] D. Bardin et al., Z. Phys. C 44 (1989) 493; Comput. Phys. Commun. 59 (1990) 303.

[9] DELPHI Collab., P. Abreu et al., Phys. Lett. B 247 (1990) $167 ;$ B 252 ( 1990$) 149$.

[10] F. Dydak, Results from LEP and SLC, preprint CERN-PPE/ 90-14 (1990).

[11] Particle Data Group. J.J. Hernández et al., Review of particle properties, Phys. Lett. B 239 (1990) 1.

[12] DELPHI Collab., P. Abreu et al., Phys. Lett. B 241 (1990) 435

[13] T. Sjöstrand, Comput. Phys. Commun. 28 (1983) 229.

[14] ALEPH Collab., D. Decamp et al., Phys. Lett. B 236 (1990) 511

OPAL Collab., M.Z. Akrawy et al., Phys. Lett. B 247 (1990) 448.

[15] L3 Collab., B. Adeva et al., Phys. Lett. B 251 (1990) 321.

[16] MARK II Collab., G.S. Abrams et al., Phys. Rev. Lett. 63 (1989) 2447; Phys. Rev. D 64 (1990) 1091; MARK II Collab., P.R. Burchat et al., preprint SLAC-Pub 5172 (1990). 